\title{
Literaturbericht
}

Achim Lang und Jale Tosun

\section{Policy Integration und verwandte Ansätze: Möglichkeiten der Theorieintegration}

\section{Einleitung*}

Folgt man einem rationalen Verständnis von Politikgestaltung, handelt es sich hierbei um einen Prozess, in dessen Mittelpunkt die Lösung gesellschaftlicher Probleme steht (vgl. z. B. Scharpf 1973). Es gibt eine Reihe von Problemen, deren Lösung vergleichsweise einfach erfolgen kann. Für diese Fälle gilt, dass das vorliegende Problem ausreichend bekannt ist, und es eine staatliche Organisation gibt, welche für die Problemlösung die alleinige Zuständigkeit besitzt. Jedoch existieren auch Probleme, die komplexerer Natur sind und deren Lösung nur dann gelingen kann, wenn Akteure aus unterschiedlichen Politiksektoren in den Entscheidungs- oder Umsetzungsprozess mit einbezogen werden. Ein Beispiel hierfür stellt die Ausarbeitung von Anpassungsstrategien an den Klimawandel dar. In Deutschland führte die anhaltende politische Diskussion um dieses Thema zur Einrichtung einer interministeriellen Arbeitsgruppe, deren Mitglieder der Aufgabe nachgehen, probate Lösungsstrategien zu erarbeiten (Stecker/Mohns/Eisenack 2012).

In der policy-analytischen Literatur zu Umweltpolitik wird die Zusammenarbeit von Akteuren aus verschiedenen Politiksektoren unter der Bezeichnung „Policy Integration“ diskutiert (vgl. z. B. Böcher/Töller 2012; Hubo/Krott 2007; Jänicke/Jörgens 2004). Diese Forschungsperspektive verweist in erster Linie auf die Schwierigkeiten, die sich bei der Formulierung und Umsetzung von sektorübergreifenden Policies ergeben können. Die Hauptargumentationslinie besteht darin, dass die sek-

* Die Autoren bedanken sich bei Dietmar Braun, Christiane Hubo, Thurid Husted, Max Krott, Johannes Marx, Philipp Trein und Sylvia Veith für hilfreiche Anmerkungen und Verbesserungsvorschläge zu früheren Versionen des Manuskripts, die 2013 auf der Jahrestagung der DVPW-Sektion PolicyAnalyse und Verwaltungswissenschaft in Bamberg und der ECPR General Conference in Bordeaux vorgestellt wurden. Susanna Killmayer und Dorothea Winter gebührt Dank für die Unterstützung bei der Literaturrecherche. 
torspezifischen Bürokratien und deren Klientel sowie die institutionellen Eigenheiten der Politiksektoren einer erfolgreichen Policy Integration potenziell entgegenwirken (vgl. Giessen u. a. 2013: 264).

Wie dieser Beitrag zeigen wird, gibt es weitere Ansätze in der politik- und verwaltungswissenschaftlichen Literatur, die sich mit der sektorübergreifenden Koordination von Regierungs- und Verwaltungshandeln befassen (vgl. auch Hustedt/ Veit 2014). Ebenso wie das Konzept der Policy Integration vor allem in Untersuchungen zur Umweltpolitik anzutreffen ist, werden die anderen Ansätze in verschiedenen sektorspezifischen Literatursträngen verwendet. Hieraus ergibt sich eine starke Fragmentierung der Forschungsliteratur, was eine stärkere theoretische Durchdringung des Untersuchungsgegenstands bislang verhindert hat. Daher verfolgt dieser Beitrag nicht nur das Ziel, einen systematischen Überblick über die Forschungsliteratur zu geben, sondern vergleicht auch die den verschiedenen Ansätzen inhärenten theoretischen Aussagen, um auf diese Weise die Möglichkeit einer Theorieintegration zu erörtern.

Die folgenden Kapitel werden zunächst erläutern, welche Voraussetzungen erfüllt sein müssen, damit Theorien miteinander verglichen und integriert werden können und was die empirische Grundlage der Abhandlung ist. Danach werden die verschiedenen Ansätze vorgestellt und ihre Theoriegerüste einem Vergleich unterzogen. Aus dieser Diskussion werden die Möglichkeiten der Theorieintegration abgeleitet. Schließlich werden die wichtigsten Befunde zusammengetragen und Perspektiven für zukünftige Forschungsarbeiten aufgezeigt.

\section{Grundlagen von Theorievergleich und -integration}

Das zentrale Anliegen dieses Beitrags ist die Skizzierung der verschiedenen Literaturstränge, die sich mit der Frage befassen, wie sektorübergreifende Policies formuliert und umgesetzt werden. Allerdings soll die Darstellung nicht rein deskriptiver Natur sein, sondern vielmehr aufzeigen, wie die verschiedenen theoretischen Ansätze prinzipiell integriert werden können, um so den Weg für eine ,integrierte“ Literatur zu Policy Integration zu bereiten. Wir folgen dabei der Empfehlung von Michael Schmid, der eine Notwendigkeit des Theorievergleichs in Situationen betont, ,wenn es mehrere Theorieangebote gibt, aber zunächst unklar ist, in welchem logischen Verhältnis diese zueinander stehen, und damit, in welchem Umfang sich ihre Erklärungsleistungen gleichen oder aber unterscheiden" (Schmid 2009: 333, Hervorhebung im Original). Mithilfe des Theorievergleichs wollen wir der Frage nachgehen, ob die Theorienangebote in der fragmentierten Literatur zu Policy Integration kommensurabel und kompatibel sind. Hierbei gilt, dass Theorien kom- 
Policy Integration und verwandte Ansätze: Möglichkeiten der Theorieintegration

mensurabel sind, wenn sie über gemeinsame Prädikate (theoretische Begriffe) oder kausale Aussagen verfügen. Kompatibilität ist dann gegeben, wenn die zu vergleichenden Theorien Prädikate besitzen, die einen gemeinsamen oder sich deckenden Werte- oder Bedeutungsbereich besitzen und sich ihre Aussagen und Folgerungen nicht widersprechen (ebd.).

Der Vergleich der theoretischen Ansätze im Hinblick auf Kommensurabilität und Kompatibilität liefert Erkenntnisse darüber, ob sich diese integrieren lassen. Je nachdem, wie sich das Verhältnis zwischen den theoretischen Ansätzen bezogen auf diese beiden Dimension darstellt, ergeben sich unterschiedliche Möglichkeiten der Theorieintegration (vgl. Jupille/Caporaso/Checkel 2003; Liska/Krohn/Messner 1989):

- Die deduktive Integration stellt die erste Möglichkeit dar und bezieht sich auf eine Situation, in der eine Theorie durch eine andere vereinnahmt werden kann. Folgt man diesem Modell, kann die Theorie mit dem kleineren empirischen Geltungsbereich als spezielle Theorie unter die allgemeinere Theorie mit dem größeren Geltungsbereich subsummiert werden. Für theoretische Ansätze, die deduktiv integrierbar sind, gilt, dass sie kommensurabel und kompatibel sind.

- Das Kennzeichen der sequentiellen Integration ist, dass Theorien in eine logisch konsistente Reihenfolge gebracht werden. Das Explanandum einer Theorie ist dann das Explans einer anderen Theorie. Im Gegensatz zur deduktiven Integration sind sequentiell integrierte theoretische Ansätze nur teilweise kommensurabel und kompatibel.

- Die horizontale Integration ordnet die Theorien nach ihrem empirischen Geltungsbereich. In diesem Fall haben alle Theorien das gleiche Explanandum, aber unterschiedliche Geltungsbereiche. Im Falle der Policy Integration wären verschiedene Politikfelder als unterschiedliche Geltungsbereiche einer Theorie anzusehen. Horizontal integrierbare theoretische Ansätze sind kommensurabel, aber nicht kompatibel.

In den nachfolgenden Abschnitten werden die Kommensurabilität und Kompatibilität der in der Forschungsliteratur verwendeten theoretischen Ansätze untersucht, um auf diese Weise die geeignete Form der Theorieintegration - deduktiv, sequentiell oder horizontal - zu ermitteln. 


\section{Erläuterungen zur Literaturbasis}

Die empirische Grundlage dieser Abhandlung sind wissenschaftliche Arbeiten, die sich mit Policy Integration und verwandten Ansätzen befassen. Um die Literatur in möglichst systematischer Form zu erfassen, wurde ein Schneeballverfahren angewandt, als dessen Startpunkt die Literatur zur Policy Integration diente. Durch eine anschließende Zitationsanalyse konnten verwandte theoretische Ansätze identifiziert werden. Als besonders einfach erwies es sich, ausgehend von Policy Integration zu den theoretischen Ansätzen ,joined-up-government“ (JUG), ,whole-of-government“ (WOG) und „Policy Kohärenz“ zu gelangen. Diese Schlagwörter wurden in die Fachdatenbank Web of Science und in die Suchmaschine Google Scholar eingegeben, um auf weitere einschlägige Ansätze zu stoßen. Insgesamt hat diese Recherche zu zahlreichen Treffern geführt, die in einem nächsten Schritt gefiltert werden mussten, um nicht den Rahmen dieses Beitrags zu sprengen. Als Ausschlusskriterium diente, dass es sich bei allen zu berücksichtigenden Ansätze um solche handeln musste, die sich mit Politikformulierung und -umsetzung im sektorübergreifenden Kontext befassen. Als Konsequenz hieraus werden die Literatur zu „Policy-Planung“, „Policy-Konsistenz“, „,komplexen Policies“ bzw. „,interdependenten Policies“, „horizontalem Regieren“" sowie Arbeiten zu Governance nicht berücksichtigt.

Tabelle 1 präsentiert die Ansätze, die für die Literatur-Analyse in Betracht gezogen wurden. Die Bezeichnung der theoretischen Ansätze ist in deutscher und englischer Sprache angegeben bzw. es wird die gebräuchliche Abkürzung aufgeführt. Die letzte Spalte enthält Informationen über die Anzahl der Artikel, die im Web of Science zu den jeweiligen Ansätzen gefunden wurden. Die Tabelle verdeutlicht, dass die Ansätze „Umfassende Planung“ und „Policy Integration“ die Literatur dominieren. Allerdings gibt es auch eine große Anzahl an Arbeiten, die sich auf die Ansätzen des JUG und WOG sowie Policy Kohärenz beziehen. Weniger prominent vertreten sind Untersuchungen zu „sektorübergreifenden Policy-Regimen“ und „horizontaler Governance“. Besonders klein hingegen ist die Anzahl an Studien, die die Ansätze ,ganzheitliche Governance“ und ,ganzheitliches Regieren“verwenden, so dass hier von keinem gut entwickelten Literaturstrang gesprochen werden kann. Daher beschränkt sich die nachfolgende Analyse auf die sieben gebräuchlichsten Ansätze und lässt die Literatur zu ganzheitlicher Governance und ganzheitlichem Regieren außen vor. 
Policy Integration und verwandte Ansätze: Möglichkeiten der Theorieintegration

Tabelle 1: Übersicht über die Literaturbasis

\begin{tabular}{llc}
\hline Ansätze & $\begin{array}{l}\text { Englische Bezeichnung oder } \\
\text { gebräuchliche Abkürzung }\end{array}$ & $\begin{array}{c}\text { Gesamtzahl der Arti- } \\
\text { kel im Web of Science }\end{array}$ \\
\hline Umfassende Planung & Comprehensive planning & 382 \\
Policy Integration & Policy integration & 286 \\
Whole-of-government & WOG & 111 \\
Policy Kohärenz & Policy coherence & 110 \\
Joined-up-government & JUG & 63 \\
Sektorübergreifende Policy-Regime & Boundary-spanning policy regimes & 23 \\
Horizontale Governance & Horizontal governance & 14 \\
Ganzheitliches Regieren* & Holistic government & 4 \\
Ganzheitliche Governance* & Holistic governance & 3 \\
\hline
\end{tabular}

Anmerkungen: Literaturstand vom 11. August 2014. * Diese Ansätze werden nicht weiter diskutiert.

Weiter sei darauf hingewiesen, dass die deutschsprachige und durch die Arbeit von Fritz W. Scharpf geprägte Literatur zu Planung als politischem Prozess nicht in dieser Abhandlung berücksichtigt wird (Scharpf 1973, 1978). Zwar enthielt diese Literatur bereits einige der hier behandelten theoretischen Prädikate, doch ging es Scharpf bei seiner Arbeit in erster Linie darum, zu verdeutlichen, dass Politik nicht nur als ein Prozess der Aufgabenerfüllung verstanden werden kann, sondern gleichfalls einen konfliktreichen Prozess darstellt, bei dem es um verschiedenartige Interessen von Akteuren geht. Auch die theoretischen Arbeiten von Scharpf und Mayntz nehmen einige Koordinationsformen der hier untersuchten Ansätze vorweg (Mayntz/Scharpf 1973). Allerdings steht bei der Unterscheidung zwischen positiver und negativer Koordination insbesondere die Politikformulierung in föderalen Systemen im Vordergrund, was auch darin zum Ausdruck kommt, dass dieser theoretische Zugang schlussendlich in der Debatte über die Politikverflechtung auf europäischer Ebene und deren Konsequenzen für die Politikgestaltung in den Mitgliedstaaten aufgegangen ist (Scharpf 1988). Diese wenngleich instruktive Literatur ist jedoch nicht Gegenstand der vorliegenden Abhandlung.

Die nachfolgende Analyse soll aufzeigen, in welchem logischen Verhältnis die theoretischen Ansätze zueinander stehen und ob sie sich für eine Theorieintegration eignen. Hierfür werden für die sieben zu besprechenden Ansätze - sofern vorhanden - in erster Linie Überblicksartikel herangezogen. Es kann bereits vorab konstatiert werden, dass sich die Ansätze stark darin unterscheiden, wie ausgeprägt ihr theoretisches Fundament ist. Da sich die Begriffsgenese bei einigen Ansätzen nicht im Wissenschaftssystem vollzog, sondern von politischen Akteuren wie der Europäischen Kommission oder nationalen Regierungen vorangetrieben wurde, weisen die betreffenden Ansätze eine erkennbar präskriptive Ausrichtung auf, wie dies vor 
allem bei der Literatur zu $J U G$ und $W O G$ der Fall ist. Zudem sei angemerkt, dass die meisten Ansätze inhaltlich durchaus heterogen sind.

Trotz des teilweise beachtlichen Volumens der Forschungsliteratur ist der Theoretisierungsgrad der hier präsentierten Ansätze nicht so weit fortgeschritten, dass von Theorien im engeren Sinne gesprochen kann. Vielmehr handelt es sich um analytische Gerüste, die dazu dienen, empirische Tatbestände zu erfassen und zu ordnen (Mayntz/Scharpf 1995: 39). Sie stellen somit kein System von Aussagen zur Erklärung empirischer Phänomene dar, sondern zeichnen sich durch eine implizite Theoretisierung aus. Nichtsdestotrotz weisen die Ansätze analytische Elemente auf, die anzeigen, dass sie darauf abzielen, kausale Verbindungen zwischen Prädikaten herzustellen, weshalb es dennoch gerechtfertigt erscheint, von einem Theorievergleich bzw. einer Theorieintegration zu sprechen.

\section{Untersuchungsgegenstände der verschiedenen Ansätze}

Dieser Abschnitt präsentiert die Untersuchungsgegenstände und theoretischen Prädikate der einzelnen Literaturstränge. Die Reihenfolge, in der die jeweiligen Ansätze vorgestellt werden, orientiert sich daran, wie prominent sie in der Forschungsliteratur vertreten sind (vgl. Tabelle 1).

Der Ansatz der umfassenden Planung wurde bereits in den 1950er Jahren entwickelt und beruht auf einer top-down-Perspektive der Politikformulierung, in der eine zentrale (städtische) Planungsbehörde für die Durchführung eines Planungsprozesses verantwortlich ist (Dahl 1975). Der Kern dieses Ansatzes besteht in der Entwicklung eines sogenannten Masterplans, der einerseits die Detailberatungen der vollziehenden Behörde strukturiert und anleitet, andererseits den Evaluationsrahmen für die konkrete Ausgestaltung vorgibt. Zudem gibt der Masterplan auch Richtung und Umfang der Koordination zwischen den beteiligten Organisationen vor (Altshuler 1965; Friedmann 1965; Innes 1996; Kent 1964). Wie ein Masterplan erarbeitet wird und was seine Merkmale sind, stehen im Mittelpunkt der einschlägigen Forschungsarbeiten.

Der Literaturstand zu Policy Integration ist gut entwickelt und besteht in erster Linie aus empirischen Arbeiten, die sich mit Umwelt-, Nachhaltigkeits- oder Klimawandelpolitiken befassen (vgl. z. B. Böcher/Töller 2012; Giessen u. a. 2013; Jänicke/Jörgens 2004; Nilsson/Nilsson 2005). Die Dominanz von Umweltfragen in dieser Literatur rührt daher, dass der Begriff „Policy Integration“ auf den Brundtland-Bericht von 1987 zurückgeht und eng mit dem Konzept der nachhaltigen Entwicklung verknüpft ist (Lenschow/Zito 1998). Die Europäische Kommission hat diesen Begriff aufgegriffen und dessen Anwendung in der Politikgestaltung im Be- 
Policy Integration und verwandte Ansätze: Möglichkeiten der Theorieintegration

reich des Umweltschutzes vorangetrieben (Lenschow 1997). Daher ist es zulässig, bei Policy Integration von einem „europäischen“ Konzept zu sprechen, was sich auch darin manifestiert, dass zahlreiche Studien untersuchen, wie umweltpolitische Ziele mit den Zielen von EU-Policies in anderen Sektoren (z. B. Energiepolitik) verknüpft werden können (Tosun/Solorio 2011).

Allgemein stehen zwei Untersuchungsgegenstände im Zentrum dieser Forschungsperspektive (Jordan/Lenschow 2010). Zum einen wird Policy Integration als ein Prozess definiert, in dem die Belange eines Politikfeldes in den Entscheidungsprozessen eines anderen Politikfeldes berücksichtigt werden (Hertin/Berkhout 2003). Dieser Aspekt deckt sich mit den Untersuchungsgegenständen der stärker verwaltungsbezogenen Ansätze $J U G$ und $W O G$ und beinhaltet Fragen nach dem Vorhandensein von interministeriellen Koordinierungsgremien und Koordinationsinstrumenten. Zum anderen wird Policy Integration in der Literatur als ein ,ideales“ Politikergebnis diskutiert, also eines, dass Policies hervorbringt, deren sektorspezifische Zielvorstellungen sich nicht widersprechen, sondern bestenfalls sogar wechselseitig verstärken (Jordan/Lenschow 2010). Vor allem die Forschungsarbeiten zu dieser zweiten Perspektive sind anschlussfähig an den theoretischen Ansatz der Policy Kohärenz.

Policy Kohärenz kam ursprünglich in den späten 1990er Jahren auf und wurde besonders durch internationale Organisationen wie die Vereinten Nationen, die Organisation für wirtschaftliche Zusammenarbeit und Entwicklung (OECD) oder die Europäische Union gefördert. Konkret geht es bei diesem Ansatz darum, dass Inkohärenzen aus unterschiedlichen Zielsetzungen verschiedener sektoraler Policies beseitigt werden sollen. Ein Beispiel sind Exporte von Überschuss-Agrarprodukten der Europäischen Union in Entwicklungsländer zu Niedrigpreisen, die der EU-Entwicklungszusammenarbeit mit dem Ziel der Sicherung der dortigen Ernährungssituation entgegenlaufen (Obrovsky 2007: 4). Aufgrund seiner Entstehung in der Praxis hat dieser Ansatz anfangs kein nennenswertes theoretisches Fundament aufgewiesen. Dieses wurde erst im Zuge der US-amerikanischen Forschung zu PolicySubsystemen und -Regimen entwickelt (May u. a. 2005: 55), das Policy Kohärenz in Verbindung mit gemeinsamen Ideen und Zielen von sektoralen Akteuren bringt (May/Sapotichne/Workman 2006: 381).

Die nächsten beiden Ansätze - JUG und $W O G$ - haben als zentralen Untersuchungsgegenstand das koordinierte und integrierte Verwaltungshandeln. Die Ansätze sind in theoretischer Hinsicht eng miteinander verwandt, werden aber auf verschiedene Policy-Bereiche angewandt. Der Ausgangspunkt beider Ansätze ist die Frage nach den Auswirkungen der Einführung des New Public Management auf den britischen und australischen Verwaltungsapparat. Durch die Reformen wurden 
kleinteilige Verwaltungsstrukturen geschaffen, die durch das Ergreifen geeigneter Maßnahmen wieder integriert werden sollten, um eine bessere Bereitstellung von staatlichen Dienstleistungen zu gewährleisten. Die Art der Integration und Koordination von fragmentierten Verwaltungsstrukturen stellt somit den Untersuchungsgegenstand der Literatur JUG und WOG dar (Pollitt 2003).

Der $J U G$-Ansatz entwickelte sich im Zuge der britischen Verwaltungsmodernisierung, die mit dem Bericht „Wiring it up: Whitehall's Management of Cross Cutting Policies and Services" angestoßen wurde (Performance and Innovation Unit 2000). Die JUG-Agenda war Teil von New Labours ambitioniertem Reformprogramm „Modernising Government” (Cabinet Office 1999) und ausdrücklich als Problemlösungsstrategie konzipiert, in der eine verstärkte Koordinierung zwischen Ministerien und nachgelagerten Behörden eine zentrale Rolle einnimmt (Clark 2002). In der Literatur werden verschiedene $J U G$-Instrumente genannt (Ling 2002):

- Neue Organisationstypen, die flexible Organisationsstrukturen und weniger introspektive Handlungsschemata verlangen.

- Neue Arten der Zusammenarbeit zwischen Organisationen, die auf gemeinsame Führungsstrukturen, gemeinsame Budgets, zusammengelegte Strukturen und gemeinsame Teams fokussiert sind.

- Neue Arten der Leistungserbringung, die eine Bündelung staatlicher Dienstleistungen aus der Perspektive des Bürgers erfordern.

Der WOG-Ansatz wird seit Mitte der 2000er Jahre zunehmend aufgegriffen, um intersektorale Koordinierungsprobleme vorwiegend im Bereich der Gesundheitsund Umweltpolitik zu analysieren. Ausgangspunkt ist wie schon beim $J U G$-Ansatz die Versäulung und fehlende Koordination der öffentlichen Verwaltung in den meist angelsächsischen - Ländern, die sich besonders der Umsetzung des New Public Management verschrieben haben (Christensen/Lagreid 2007).

Der Ansatz der sektorübergreifenden Policy-Regime befasst sich mit Politikproblemen, deren Lösungen besondere Formen der interinstitutionellen Koordinierung und Steuerung bedürfen (May/Jochim/Sapotichne 2011). Diese Formen der institutionellen Koordinierung bilden den Untersuchungsgegenstand der korrespondierenden Literatur. Der Ansatz der sektorübergreifenden Policy-Regime kann als Erweiterung von Policy Kohärenz betrachtet werden, bei dem nicht die Policies an sich im Vordergrund stehen, sondern die Strukturen und Institutionen, die eine Kohärenz in den Policies bzw. Policy-Zielen bewirken sollen (Jochim/May 2010; May/ Jochim/Sapotichne 2011).

Der Ansatz der horizontalen Governance versteht sich als Extension der Ansätze $J U G$ und WOG (Murray/Fagan/McCusker 2009; Termeer 2009). Die Literatur zu horizontaler Governance besteht in erster Linie aus Arbeiten, die Vorschläge un- 
terbreiten, auf welche Weise die Erbringung staatlicher Dienstleistungen erfolgen sollte. Grundsätzlich empfiehlt diese Literatur, hierarchische Steuerungselemente möglichst umfassend durch horizontale Netzwerke zwischen verschiedenen Regierungs- und Verwaltungseinheiten sowie zivilgesellschaftlichen Akteuren zu ersetzen.

Tabelle 2 fasst die Untersuchungsgegenstände der sieben Ansätze zusammen. So wird ersichtlich, dass sich fünf der Ansätze mit interorganisatorischer bzw. -institutioneller Koordination befassen. Während sich die Ansätze des JUG und WOG auf die Dimension der administrativen Koordination und Integration beschränken, beziehen horizontale Governance, Policy Integration und sektorübergreifende Policy-Regime auch die Interessen nichtstaatlicher Akteuren mit in ihre konzeptionellen Überlegungen ein. Umfassende Planung und Policy Kohärenz hingegen unterscheiden sich von den übrigen Ansätzen dahingehend, dass bei ihnen die konkrete Ausgestaltung von Policies im Vordergrund steht und nicht so sehr der Politikgestaltungs- bzw. Umsetzungsprozess. Zuletzt sei darauf verwiesen, dass die verschiedenen Ansätze in Untersuchungen von jeweils verschiedenen Politiksektoren angewandt werden, wie dies aus der dritten Spalte von Tabelle 2 hervorgeht.

Tabelle 2: Übersicht über die Untersuchungsgegenstände

\begin{tabular}{|c|c|c|}
\hline Ansätze & Untersuchungsgegenstände & Politikfelder \\
\hline Umfassende Planung & Erarbeitung eines Masterplans & Lokale Politik \\
\hline Policy Integration & $\begin{array}{l}\text { Interorganisationelle Zusammenarbeit und Ko- } \\
\text { ordination; Berücksichtigung von Zielen anderer } \\
\text { Politikfelder }\end{array}$ & $\begin{array}{l}\text { Umwelt- und Klimawandel- } \\
\text { politiken }\end{array}$ \\
\hline Policy Kohärenz & $\begin{array}{l}\text { Policies, die gemeinsame Ziele und Ideen verfol- } \\
\text { gen }\end{array}$ & $\begin{array}{l}\text { Internationale Entwicklungs- } \\
\text { hilfepolitik }\end{array}$ \\
\hline $\begin{array}{l}\text { Whole-of-government } \\
\text { (WOG) }\end{array}$ & $\begin{array}{l}\text { Koordiniertes und integriertes Verwaltungshan- } \\
\text { deln }\end{array}$ & Verwaltungsreform \\
\hline $\begin{array}{l}\text { Joined-up-government } \\
\text { (JUG) }\end{array}$ & $\begin{array}{l}\text { Koordiniertes und integriertes Verwaltungshan- } \\
\text { deln }\end{array}$ & $\begin{array}{l}\text { Verwaltungsreform, Gesund- } \\
\text { heitspolitik }\end{array}$ \\
\hline $\begin{array}{l}\text { Sektorübergreifende Poli- } \\
\text { cy-Regime }\end{array}$ & Interinstitutionelle Koordinierung & $\begin{array}{l}\text { Lokale und einzelne nationa- } \\
\text { le Politiken }\end{array}$ \\
\hline Horizontale Governance & $\begin{array}{l}\text { Entwicklung von horizontalen Koordinierungs- } \\
\text { strukturen }\end{array}$ & Lokale Politik \\
\hline
\end{tabular}

\section{Theoretische Argumente der einzelnen Ansätze}

Die Literatur zur umfassenden Planung legt den Fokus auf die Erstellung eines städtischen Masterplans und untersucht den Einfluss von Stakeholder-Beteiligung und zentraler Steuerung durch Budgetierung und Überwachung als entscheidende Faktoren in der Koordination des Planungsprozesses (Glasbergen 1992; Mostert 
2006; Sanchirico u. a. 2010). Somit ist die Interaktion von staatlichen und nichtstaatlichen Akteuren von großer Bedeutung für diesen Literaturstrang.

Der Ansatz der Policy Integration berücksichtigt verschiedene Erklärfaktoren für eine erfolgreiche Integration sektorübergreifender Policies. So argumentieren beispielsweise Nilsson und Nilsson (2005) bezüglich der Integration von Klimawandel-Policies, dass diese nur dann mit Maßnahmen aus angrenzenden Politikfeldern integriert werden können, wenn das zugrunde liegende Politikproblem so dargestellt werden kann, dass sich alle sektorspezifischen Akteure hiervon betroffen und verantwortlich für die Problemlösung fühlen. Jordan und Lenschow (2010) identifizieren in ihrem Literaturbericht weitere potenzielle Erfolgsfaktoren für Policy Integration. Dazu zählen institutionelle Faktoren wie der Grad der ministeriellen Ressortunabhängigkeit und die Ausgestaltung der föderalen Staatsstrukturen. Auch eine legalistische Verwaltungskultur ist laut den Autoren für eine gelungene Policy Integration eher hinderlich. Zudem argumentieren Jordan und Lenschow, dass Interessenkonstellationen Auswirkungen auf die Kooperations- und Integrationsbereitschaft im Rahmen von Policy Integration haben. Vor diesem Hintergrund stellen sie die Erwartung auf, dass Mitte-Links-Regierungen Befürworter einer Integration von Policies sind, während Mitte-Rechts-Regierungen eher auf klare Ressortzuständigkeiten setzen und somit eine geringere Präferenz für Policy Integration besitzen.

Die Literatur zu Policy Kohärenz hebt in analytischer Hinsicht drei Faktoren hervor: die Eigenschaft der betroffenen Politikbereiche, die Anzahl der betroffenen Interessen und die Existenz von integrierenden Institutionen (May u. a. 2005; May/ Sapotichne/Workman 2006). Im Hinblick auf die Politikbereiche wird zwischen substanziellen (z. B. Umwelt oder Gesundheit), gruppenspezifischen (z. B. Familien oder Senioren) und räumlichen Politikbereichen (lokal, regional oder bundesweit) unterschieden. Die einschlägigen Forschungsbeiträge argumentieren, dass Policy Kohärenz in substanziellen Politikbereichen höher, jedoch in Politikbereichen mit einer hohen Problemdichte geringer ist, da die politische Aufmerksamkeit auf viele verschiedene Problembereiche streut. Es kommt also nicht nur darauf an, wie groß der Betroffenenkreis ist, sondern auch, wie gut er sich auf eine bestimmte Problemlage konzentrieren kann. Als letzter Punkt ist zu nennen, dass in der Literatur die These vertreten wird, dass das Vorhandensein integrierender Institutionen der Kohärenz von Policies zuträglich ist, da diese dazu beitragen, die Aufmerksamkeit der am Formulierungsprozess beteiligten Akteure zu fokussieren.

$J U G$ und $W O G$ befassen sich mit interadministrativer Koordination und wurden in jüngerer Zeit derart weiterentwickelt, dass sie nun auch ein theoretisches Fundament besitzen. Vor allem die Literatur zu $J U G$ betont die Wichtigkeit von Ver- 
Policy Integration und verwandte Ansätze: Möglichkeiten der Theorieintegration

trauen zwischen den beteiligten Organisationen und Personen für eine erfolgreiche organisationsübergreifende Zusammenarbeit (O’Flynn u. a. 2011). So müssen organisatorische Veränderungen durch kulturelle Maßnahmen flankiert werden, um eine kooperative und an interorganisatorischen Problemen orientierte Organisationskultur zu fördern (Halligan 2007). In diesem Zusammenhang argumentieren etwa Davies (2009) und Friend (2006), dass insbesondere Interessengegensätze zwischen den beteiligten Verwaltungseinheiten eine erfolgreiche Zusammenarbeit verhindern können. Die Schaffung von speziellen Anreizen soll hier die Bereitschaft zur Zusammenarbeit erhöhen. Untersuchungen, die sich mit WOG und JUG befassen, heben zudem den positiven Effekt von Leistungs- und Output-Kennzahlen hervor (Boston/Pallot 1997).

Die Arbeiten zu sektorübergreifenden Policy-Regimen bestechen vor allem dadurch, dass sie eine theoretische Begründung für die Integration von Policies liefern, was sie anschlussfähig an die Literatur zu Policy Integration, aber vor allem zu Policy Kohärenz macht. So betonen beide Ansätze - sektorübergreifende PolicyRegime und Policy Kohärenz -, dass integrierende Institutionen von großer Bedeutung sind. Unter integrierenden Institutionen subsummieren May, Jochim und Sapotichne (2011) Parlamentsausschüsse und regierungsnahe Beratungsgremien. Die Autoren weisen neben integrierenden Institutionen noch auf drei weitere Einflussfaktoren hin, die die Kontur eines sektorübergreifenden Policy-Regimes bestimmen können. Der erste Einflussfaktor sind Policy-Probleme, die einerseits die Aufmerksamkeit der Akteure auf sich ziehen und andererseits bereits integrierend auf die bestehenden Akteurskonstellationen einwirken. Den zweiten Einflussfaktor stellen Policy-Vorstellungen (,,ideas“) dar, die von den Autoren als politische Festlegungen und Verpflichtungen verstanden werden, welche die Richtung der politischen Aktivitäten der beteiligten Akteure bestimmen. Der dritte Einflussfaktor sind Interessen, die das Ausmaß an Konsens und Konflikt innerhalb eines Policy-Subsystems anzeigen. Es wird argumentiert, dass Unterschiede in der Problemperzeption, den Policy-Vorstellungen sowie das Fehlen von integrierenden Institutionen die Stabilität von sektorübergreifenden Policy-Regimen verringern (Jochim/May 2010; May/ Jochim/Sapotichne 2011).

Die Literatur zur horizontalen Governance liefert einige Erklärfaktoren für die Wirksamkeit von integrierenden Politikinstrumenten. So erhöht laut Smismans (2008) die Zusammenarbeit mit zivilgesellschaftlichen Akteuren einerseits die Legitimität von Policy-Ergebnissen. Andererseits werden Entscheidungen über die Wahl der Politikinstrumente verzögert und die Wahrscheinlichkeit nimmt zu, dass es zu keinem Konsens über Ziele und Mittel kommt. Auch weist die Literatur darauf hin, dass ein partnerschaftliches Verhältnis zwischen zivilgesellschaftlichen und 
staatlichen Akteuren häufig an den Einstellungen und internen Entscheidungsregeln staatlicher Organisationen scheitert (Termeer 2009). In diesem Zusammenhang erhöht sich wiederum die Bedeutung eines zentralen und mit Durchsetzungsrechten ausgestatteten Koordinierungsorgans, das hierarchisch steuert (Phillips 2004: 395), wodurch aus der horizontalen Governance schlussendlich wieder ein staatszentrierter Ansatz wird (Flinders 2002).

Tabelle 3 trägt die Erklärfaktoren zusammen, die aus den verschiedenen Literatursträngen abgeleitet werden konnten.

Tabelle 3: Überblick über die Erklärfaktoren

\begin{tabular}{|c|c|}
\hline Konzept & Erklärfaktoren \\
\hline Umfassende Planung & $\begin{array}{l}\text { Zentrale Steuerung (Budgetierung, Monitoring), Beteiligung von Stakehol- } \\
\text { dern }\end{array}$ \\
\hline Policy Integration & $\begin{array}{l}\text { Verantwortlichkeit/Betroffenheit der Akteure, ministerielle Unabhängigkeit, } \\
\text { Föderalismus, Parteiendifferenz, Verwaltungskultur }\end{array}$ \\
\hline Policy Kohärenz & $\begin{array}{l}\text { Art des Problems } \rightarrow \text { homogene Interessen und Problemperzeption, integrie- } \\
\text { rende Institutionen }\end{array}$ \\
\hline $\begin{array}{l}\text { Whole-of-Government } \\
\text { (WOG) }\end{array}$ & $\begin{array}{l}\text { Zurechenbarkeit der Leistung (Kennzahlen), Anreize für Zusammenarbeit, } \\
\text { Vertrauen }\end{array}$ \\
\hline $\begin{array}{l}\text { Joined-up-Government } \\
\text { (JUG) }\end{array}$ & $\begin{array}{l}\text { Zurechenbarkeit der Leistung (Kennzahlen), Anreize für Zusammenarbeit, } \\
\text { Vertrauen }\end{array}$ \\
\hline $\begin{array}{l}\text { Sektorübergreifende Policy- } \\
\text { Regime }\end{array}$ & $\begin{array}{l}\text { Integrierende Institutionen (z. B. Ausschüsse), Homogenität von Interessen, } \\
\text { geteilte Ideen und Problemperzeption }\end{array}$ \\
\hline Horizontale Governance & $\begin{array}{l}\text { Zusammenarbeit zivilgesellschaftlicher Akteure erhöht die Legitimation, aber } \\
\text { verzögert die Entscheidungsfindung; zentrales Koordinierungsorgan behebt } \\
\text { Entscheidungsblockaden }\end{array}$ \\
\hline
\end{tabular}

\section{Integration der verschiedenen theoretischen Ansätze}

Die Diskussion im vorangegangenen Abschnitt hat wichtige Erkenntnisse darüber geliefert, wie sich die verschiedenen Ansätze zueinander verhalten. Als erstes lässt sich feststellen, dass die Ansätze kommensurabel sind, da sie gemeinsame Prädikate aufweisen. Besonders deutlich wird dies im Bereich der Koordinationsinstrumente. Die meisten Ansätze betonen die Schaffung oder Zusammenführung von Organisationen und die Einbindung von Stakeholdern. Die Ansätze sind allerdings häufig nicht kompatibel, da die Prädikate einen unterschiedlichen Werte- bzw. Gegenstandsbereich umfassen. Dies überrascht nicht, da die meisten Ansätze in unterschiedlichen Politiksektoren entstanden sind oder ausschließlich bei der Analyse bestimmter sektoraler Policies angewandt werden (vgl. Tabelle 2), so dass nicht bestimmt werden kann, wie die Prädikate implizit durch sektorspezifische Argumente beeinflusst sind. 
Wie eingangs dargestellt, besteht grundsätzlich die Möglichkeit, Theorien entweder deduktiv, sequenziell oder horizontal zu integrieren. JUG und WOG sind sich in ihrem Erkenntnisinteresse sehr ähnlich, so dass sie leicht integriert werden können. Diese Ansätze sind zeitlich nacheinander entstanden und haben sich gegenseitig rezipiert. Sie teilen gemeinsame Prädikate und haben mit der angelsächsischen Verwaltungspraxis einen ähnlichen Geltungsbereich. Sie sind deshalb kommensurabel und kompatibel. Dadurch können diese beiden Ansätze deduktiv integriert werden. Ein integriertes Modell könnte noch um den Ansatz der horizontalen Governance erweitert werden, da dieser eine Weiterentwicklung von JUG und WOG um horizontale Netzwerke darstellt. Allerdings ist der Geltungsbereich von horizontaler Governance ein anderer, so dass es sich hierbei um eine horizontale Integration handeln würde. Für eine deduktive Integration bieten sich zudem Policy Kohärenz und Policy Integration an. Hierbei würde die Policy Kohärenz den Ansatz mit dem spezielleren Geltungsbereich darstellen, der unter Policy Integration subsumiert wird.

Den Ausgangspunkt für eine sequenzielle Theorieintegration bietet der Ansatz der Policy Kohärenz. Die Formulierung einer kohärenten Policy würde in einem sequentiell integrierten Modell die abhängige Variable darstellen, die kausal durch das Vorhandensein und die Ausprägung von Koordinations- und Integrationsmaßnahmen bestimmt ist. Ein ähnlicher Vorschlag findet sich bei Jordan und Lenschow (2010), doch die Autoren konzentrieren sich in ihren Ausführungen ausschließlich auf den Ansatz der Policy Integration und streben eine theoretische Stärkung konkret dieses Ansatzes anstatt einer Integration verschiedener theoretischer Ansätze an.

Wie Abbildung 1 zeigt, kann die Kohärenz von sektorübergreifenden, integrierten Policies durch zahlreiche Faktoren gefördert werden, die sich unter den Oberbegriffen „Umfang der Zusammenarbeit“ und „Grad der Koordinierung und Integration“ zusammenfassen lassen. Der Grad der Koordinierung ist als Erklärgröße den Ansätzen Policy Integration, Policy Kohärenz, umfassender Planung und sektorübergreifende Policy-Regime entnommen. In den verschiedenen Literatursträngen wird argumentiert, dass eine Zentralisierung der Führungsstruktur ebenso vorteilhaft für die Integration von Policies ist, wie ein kontinuierliches Monitoring und das Vorhandensein gemeinsamer Budgets.

Die erfolgreiche Integration von sektorübergreifenden Policies ist jedoch auch abhängig vom Umfang der Zusammenarbeit der involvierten Akteure. Die verschiedenen Ansätze - vor allem $J U G$ und $W O G$ - nennen hier unter anderem Vertrauen, Anreize für die Teilnahme an der Zusammenarbeit, geringe Interessenunterschiede der teilnehmenden Akteure und Art des Policy-Problems als wichtige 
Größe zur Bestimmung des Umfangs der Zusammenarbeit (vgl. May u. a. 2005; May/Sapotichne/Workman 2006).

Abbildung 1: Vereinfachte Darstellung von sequenzieller Integration

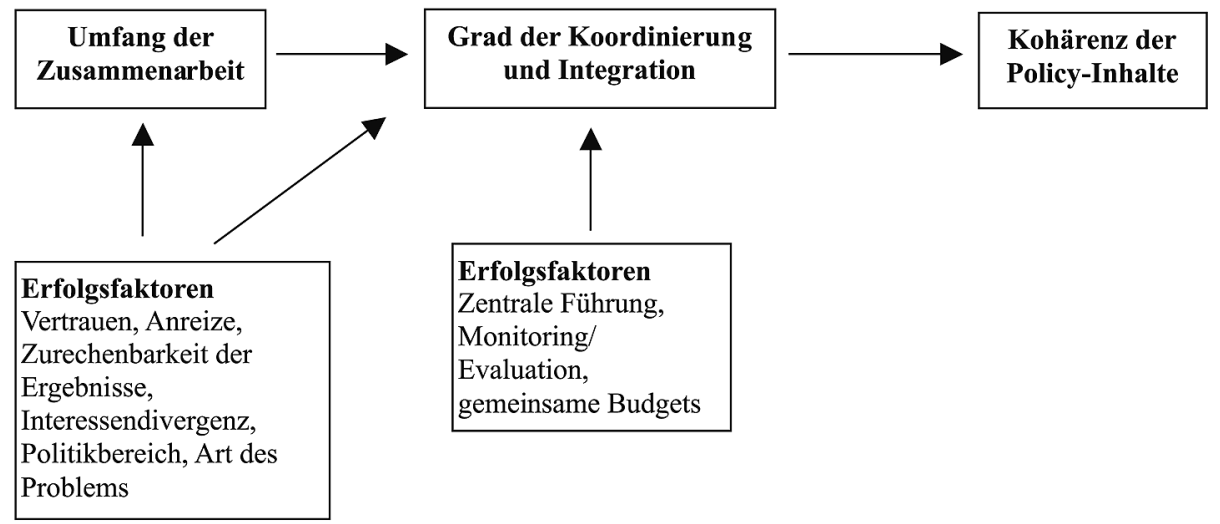

Es lässt sich somit abschließend festhalten, dass grundsätzlich Möglichkeiten der deduktiven, sequentiellen und horizontalen Integration bestehen. Damit ist eine notwendige Bedingung dafür erfüllt, dass zukünftige Forschungsarbeiten einen Beitrag dazu leisten können, eine in theoretischer Hinsicht integriertere Literatur zur Integration sektorübergreifender Policies zu schaffen.

\section{Schlussbetrachtung}

Die vorliegende Abhandlung hat einen ersten Versuch unternommen, die umfangreiche, aber fragmentierte Literatur zu Policy Integration und verwandten Ansätzen systematisch zu erfassen und die wichtigsten Merkmale der einzelnen Literaturstränge herauszuarbeiten. Auf diese Weise konnte gezeigt werden, dass es eine Reihe von Ansätzen gibt, die sich mit der Koordinierung von sektorübergreifendem Handeln in Regierung und Verwaltung befassen, die jedoch durch ihre sektorspezifische Anwendung in der Literatur bislang kaum in Dialog treten konnten.

Eine weitere Besonderheit der hier besprochenen Literatur ist, dass sie stark von empirischen Fragen dominiert ist. Die empirische Ausrichtung der Forschung rührt in erster Linie daher, dass einige der behandelten Ansätze der politischen Diskussionen in Staaten oder internationalen Organisationen entspringen. Trotz des stark ausgeprägten empirischen Charakters der diskutierten Literaturstränge konnten in diesem Beitrag Möglichkeiten aufgezeigt werden, wie die theoretischen Argumente, die den jeweiligen Ansätzen zugrunde liegen, integriert werden können. Allerdings 
Policy Integration und verwandte Ansätze: Möglichkeiten der Theorieintegration

sollen die skizzierten Möglichkeiten der Theorieintegration nicht darüber hinwegtäuschen, dass der Theoriegehalt der einzelnen Ansätze noch entwicklungsbedürftig ist. Daher erscheint es für zukünftige Forschungsanstrengungen gewinnbringend, den Fokus in erster Linie auf die theoretische Dimension zu legen. Hierzu bieten sich eine Reihe von allgemeinen Theorien aus den Bereichen Policy-Analyse, Politische Ökonomie und Verwaltungswissenschaft an.

So ist es beispielsweise denkbar, dass sektorübergreifende Policies leichter auf die Entscheidungsagenda gesetzt werden, weil sie einen größeren Adressatenkreis betreffen. Sind also sektorübergreifende Policies relativ zu anderen Policies überrepräsentiert auf der politischen Entscheidungsagenda oder doch eher unterrepräsentiert, weil sich die anschließenden Phasen der Politikformulierung und -umsetzung potenziell schwieriger gestalten? Bezogen auf diese Frage könnte prinzipiell argumentiert werden, dass die Vetomacht der einzelnen beteiligten Akteure die Verabschiedung solcher Politiken erschwert. Eine solche politikökonomische Forschungsperspektive zöge nach sich, dass die Interessen der verschiedenen Akteursgruppen bezogen auf die sektorübergreifende Policy systematisch erfasst und miteinander verglichen werden müssten. In Ansätzen wird der Einfluss der Vetoposition von Akteuren bereits in der Literatur zu Policy Integration berücksichtigt, so dass es nun gilt, diese Perspektive weiterzuentwickeln. Schließlich könnte es bei der Implementation von sektorübergreifenden Policies zu einem größeren Ausmaß an Prinzipal-Agent-Problemen kommen als bei sektorspezifischen Policies, weil gleich mehrere bürokratische Akteure mitwirken müssen und somit die Informationsasymmetrie zulasten der politischen Entscheidungsträger zunehmen sollte. Es sind solche allgemeinen theoretischen Erwartungen, die der diskutierten Literatur trotz ihres Umfangs weitgehend fehlen, aber letztendlich die Kraft besitzen, die Wissenschaftlichkeit der hier vorgestellten Ansätze zu steigern.

\section{Literatur}

Altshuler, Alan, 1965: The goals of comprehensive planning, in: Journal of the American Institute of Planners 31 (3), 186-195.

Böcher, Michael/Töller, Annette E, 2012: Umweltpolitik in Deutschland. Eine politikfeldanalytische Einführung, Wiesbaden.

Boston, Jonathan/Pallot, June, 1997: Linking strategy and performance: Developments in the New Zealand public sector, in: Journal of Policy Analysis and Management 16 (3), 382-404.

Cabinet Office, 1999: Modernising government, London. 
Christensen, Tom/Lagreid, Per, 2007: The Whole-of-Government Approach to Public Sector Reform, in: Public Administration Review 67 (6), 1059-1066.

Clark, Tim, 2002: New Labour's big idea: Joined-up government, in: Social Policy and Society 1 (02), 107-117.

Dahl, Dale C, 1975: Public Policy Changes Needed to Cope with Changing Structure, in: American Journal of Agricultural Economics 57 (2), 206-213.

Davies, Jonathan S, 2009: The limits of joined-up government: towards a political analysis, in: Public Administration 87 (1), 80-96.

Flinders, Matthew, 2002: Governance in Whitehall, in: Public Administration 80 (1), 51-75.

Friedmann, John, 1965: A response to Altshuler: comprehensive planning as a process, in: Journal of the American Institute of Planners 31 (3), 195-197.

Friend, John, 2006: Partnership meets politics: managing within the maze, in: International Journal of Public Sector Management 19 (3), 261-277.

Giessen, Lukas/Hubo, Christiane/Krott, Max/Kaufer, Ricardo, 2013: Steuerungspotentiale von Zielen und Instrumenten des Politiksektors Forstwirtschaft und deren möglicher Beitrag zu einer nachhaltigen Entwicklung ländlicher Regionen, in: Zeitschrift für Umweltpolitik \& Umweltrecht 36 (3), 261-291.

Glasbergen, Pieter, 1992: Comprehensive policy planning for water systems: the administration of complex policy networks, in: International Journal of Water Resources Development 8 (1), 45-52.

Halligan, John, 2007: Horizontal coordination in Australian government, in: Zeitschrift für Staats-und Europawissenschaften 5 (2), 203-217.

Hertin, Julia/Berkhout, Frans, 2003: Analysing institutional strategies for environmental policy integration: the case of EU enterprise policy, in: Journal of Environmental Policy \& Planning 5 (1), 39-56.

Hubo, Christiane/Krott, Max, 2007: Umsetzungsstrategien für integrative Politikansätze am Beispiel invasiver gebietsfremder Arten, in: Zeitschrift für angewandte Umweltforschung 8 (2), 216-226.

Hustedt, Thurid/Veit, Sylvia, 2014: Forschungsperspektiven auf Regierungs- und Verwaltungskoordination: Koordinationsprobleme und Erklärungsfaktoren, in: der moderne staat 7 (1), 17-36.

Innes, Judith E., 1996: Planning through consensus building: A new view of the comprehensive planning ideal, in: Journal of the American Planning Association 62 (4), 460-472.

Jänicke, Martin/Jörgens, Helge, 2004: Neue Steuerungskonzepte in der Umweltpolitik, in: Zeitschrift fur Umweltpolitik und Umweltrecht (3), 297-348. 
Policy Integration und verwandte Ansätze: Möglichkeiten der Theorieintegration

Jochim, Ashley E./May, Peter J., 2010: Beyond subsystems: Policy regimes and governance, in: Policy Studies Journal 38 (2), 303-327.

Jordan, Andrew/Lenschow, Andrea, 2010: Environmental policy integration: a state of the art review, in: Environmental Policy and Governance 20 (3), 147-158.

Jupille, Joseph/Caporaso, James A/Checkel, Jeffrey T, 2003: Integrating Institutions Rationalism, Constructivism, and the Study of the European Union, in: Comparative Political Studies 36 (1-2), 7-40.

Kent, TJ, 1964: The urban general plan, San Francisco.

Lenschow, Andrea, 1997: Variation in EC environmental policy integration: Agency push within complex institutional structures, in: Journal of European Public Policy 4 (1), 109-127.

Lenschow, Andrea/Zito, Anthony R., 1998: Blurring or Shifting of Policy Frames?: Institutionalization of the Economic-Environmental Policy Linkage in the European Community, in: Governance 11 (4), 415-441.

Ling, Tom, 2002: Delivering joined-up government in the UK: dimensions, issues and problems, in: Public Administration 80 (4), 615-642.

Liska, Allen E/Krohn, Marvin D/Messner, Steven F, 1989: Strategies and requisites for theoretical integration in the study of crime and deviance, in: Steven F Messner/Marvin D Krohn/Allen E Liska (Hrsg.), Theoretical integration in the study of deviance and crime: Problems and prospects, New York, 1-19.

May, Peter J./Jochim, Ashley/Sapotichne, Joshua, 2011: Constructing Homeland Security: An Anemic Policy Regime, in: Policy Studies Journal 39 (2), 285-307. May, Peter J./Jones, Bryan D./Beem, Betsi B./Neff-Sharum, Emily A./Poague, Melissa K., 2005: Policy Coherence and Component-Driven Policymaking: Arctic Policy in Canada and the United States, in: Policy Studies Journal 33 (1), 37-63. May, Peter J./Sapotichne, Joshua/Workman, Samuel, 2006: Policy coherence and policy domains, in: Policy Studies Journal 34 (3), 381-403.

Mayntz, Renate/Scharpf, Fritz W, 1973: Planungsorganisation: die Diskussion um die Reform von Regierung und Verwaltung des Bundes, München.

Mayntz, Renate/Scharpf, Fritz W, 1995: Der Ansatz des akteurzentrierten Institutionalismus, in: Renate Mayntz/Fritz W Scharpf (Hrsg.), Gesellschaftliche Selbstregelung und politische Steuerung, Frankfurt a. M./New York, 39-72.

Mostert, Erik, 2006: Integrated water resources management in the Netherlands: how concepts function, in: Journal of Contemporary Water Research \& Education 135 (1), 19-27.

Murray, Michael/Fagan, G/McCusker, Paul, 2009: Measuring horizontal governance: a review of public consultation by the Northern Ireland government between 2000 and 2004, in: Policy \& Politics 37 (4), 553-571. 
Nilsson, Mans/Nilsson, Lars J., 2005: Towards climate policy integration in the EU: evolving dilemmas and opportunities, in: Climate Policy 5 (3), 363-376.

Obrovsky, Michael, 2007: Entwicklungspolitische Kohärenz - Zu den erweiterten politischen Rahmenbedingungen für mehr Wirksamkeit in der Entwicklungszusammenarbeit. ÖFSE-Working Paper 16, Wien.

O'Flynn, Janine/Buick, Fiona/Blackman, Deborah/Halligan, John, 2011: You win some, you lose some: Experiments with joined-up government, in: International Journal of Public Administration 34 (4), 244-254.

Performance and Innovation Unit, 2000: Wiring it up: Whitehall's Management of Cross Cutting Policies and Services, London.

Phillips, Susan D., 2004: The Limits of Horizontal Governance, in: Society and Economy 26 (2-3), 383-405.

Pollitt, Christopher, 2003: Joined-up Government: A Survey, in: Political Studies Review 1 (1), 34-49.

Sanchirico, James M./Eagle, Josh/Palumbi, Stephen R./Thompson, Barton H., 2010: Comprehensive planning, dominant-use zones, and user rights: a new era in ocean governance, in: Bull. Mar. Sci 86 (2), 273-285.

Scharpf, Fritz W, 1973: Planung als politischer Prozeß. Aufsätze zur Theorie der planenden Demokratie, Frankfurt a. M.

Scharpf, Fritz W, 1978: Intergovernmental Policy Studies: Issues, Concepts and Perspectives, in: Kenneth Hanf/Fritz W Scharpf (Hrsg.), Interorganizational Policy Making. Limitis to Coordination and Central Control, London, 345-370. Scharpf, Fritz W, 1988: The joint-decision trap: lessons from German federalism and European integration, in: Public administration 66 (3), 239-278.

Schmid, Michael, 2009: Theorien, Modelle und Erklärungen. Einige Grundprobleme des soziologischen Theorienvergleichs, in: Gerhard Preyer (Hrsg.), Neuer Mensch und kollektive Identität in der Kommunikationsgesellschaft, Wiesbaden, 323-359.

Smismans, Stijn, 2008: New modes of governance and the participatory myth, in: West European Politics 31 (5), 874-895.

Stecker, Rebecca/Mohns, Till/Eisenack, Klaus, 2012: Anpassung an den Klimawandel - Agenda Setting und Politikintegration in Deutschland, in: Zeitschrift für Umweltpolitik und Umweltrecht 35 (2), 179-208.

Termeer, Catrien JAM, 2009: Barriers to new modes of horizontal governance: A sense-making perspective, in: Public Management Review 11 (3), 299-316.

Tosun, Jale/Solorio, Israel, 2011: Exploring the Energy-Environment Relationship in the EU: Perspectives and Challenges for Theorizing and Empirical Analysis, in: European Integration online Papers 15 (Special Mini-Issue 1), Artikel 7. 
Korrespondenzanschrift:

Dr. Achim Lang

Universität Konstanz

Fachbereich Politik- und Verwaltungswissenschaft

Universitätsstr. 10

78464 Konstanz

E-Mail: achim.lang@uni-konstanz.de

Jun.-Prof. Dr. Jale Tosun

Ruprecht-Karls-Universität Heidelberg

Institut für Politische Wissenschaft

Bergheimer Straße 58

69115 Heidelberg

E-Mail: jale.tosun@ipw.uni-heidelberg.de 